\title{
Does change in heart rate and blood pressure during adenosine stress perfusion cardiovascular magnetic resonance ( $A-C M R P)$ imaging predict perfusion defects?
}

\author{
Krishnaraj S Rathod*, Ceri Davies, Steffen E Petersen, Mark Westwood, Neha Sekhri \\ From 15th Annual SCMR Scientific Sessions \\ Orlando, FL, USA. 2-5 February 2012
}

\section{Summary}

Peripheral haemodynamic response during adenosine stress CMR perfusion does not vary among those with or without perfusion defects identified during routine clinical practice.

\section{Background}

Haemodynamic response to adenosine infusion during CMR stress perfusion continues to be used as surrogate to determine adequacy of hyperaemia in clinical setting. Our aim was to determine the proportion of patients who achieve pre-specified adequate response to adenosine (increase in heart rate (HR) by $\geq 10$ beats or a drop in systolic blood pressure (SBP) by $10 \mathrm{~mm} \mathrm{Hg}$ from baseline reading) and if this varies in patients identified with and without perfusion defect in a clinical setting.

\section{Methods}

We prospectively identified 94 consecutive patients undergoing A-CMRP, 7 of who were excluded because of missing data. 87 patients underwent first pass CMRP on a Philips Achieva CV 1.5 T MR scanner (Philips, The Netherlands), with standardised SCMR acquisition protocol infusing adenosine at $140 \mu \mathrm{g} / \mathrm{kg} / \mathrm{min}$ for $3 \mathrm{~min}$ utes with HR and SBP recorded at baseline, one, two and three minutes. All patients confirmed abstinence from caffeine and chocolate for $>24$ hours and no patients were on theophylline or dipyridamole. Descriptive statistics and logistic regression were performed.

\section{Results}

67 (77\%) patients achieved the pre-specified adequate haemodynamic response and this was mainly driven by increase in heart rate.

The OR (adjusted for age) for predicting perfusion defect by adequate haemodynamic response in patients with and without CAD was $2.5(0.58,10.40, \mathrm{p}=0.21)$ and $3.26(0.48,22.13, \mathrm{p} 0.20)$ respectively.

\section{Conclusions}

Our study shows that in patients undergoing adenosine $(140 \mu \mathrm{g} / \mathrm{kg} / \mathrm{min})$ stress perfusion, there is no significant difference in peripheral haemodynamics in patients identified with or without perfusion defect and challenges their use as a marker of hyperaemic response. It remains to be seen whether high dose adenosine will influence these findings and change clinical practice.

\section{Funding}

No funding was obtained for this study.

Published: 1 February 2012

doi:10.1186/1532-429X-14-S1-M5

Cite this article as: Rathod et al:: Does change in heart rate and blood pressure during adenosine stress perfusion cardiovascular magnetic resonance (A-CMRP) imaging predict perfusion defects? Journal of Cardiovascular Magnetic Resonance 2012 14(Suppl 1):M5. 
Table 1

\begin{tabular}{cccc}
\hline Total cohort N=87 & Adequate response N=67 (77\%) & Inadequate response N=20 (23\%) & $\mathrm{P}$ value \\
\hline Age $($ median) & $60($ IQR 47, 72) & $65($ IQR 50, 72) & 0.65 \\
Known CAD & $34(51 \%)$ & $10(50 \%)$ & 0.93 \\
Perfusion defect present & $32(48 \%)$ & $6(30 \%)$ & 0.16 \\
Good LV function $(>55 \%)$ & $58(89 \%)$ & $12(60 \%)$ & 0.01 \\
\hline
\end{tabular}

Table 2

\begin{tabular}{|c|c|c|c|}
\hline In those with known CAD N=44 & Perfusion defect present $\mathrm{N}=25(57 \%)$ & No perfusion defect $\mathrm{N}=19(43 \%)$ & $P$ value \\
\hline Age (median) & 65(IQR 50-72) & 69(IQR 51-75) & 0.69 \\
\hline Male & $22(88 \%)$ & $17(90 \%)$ & 0.88 \\
\hline Rest SBP - mean SBP over 3 minutes of adenosine & $-5.0(\mathrm{IQR}-9.0,1.0)$ & $-4.0(\operatorname{QQR}-8.0,4)$ & 0.16 \\
\hline Rest HR - mean HR over 3 minutes (beats per minute) & $13(\mathrm{IQR} 10,20)$ & $14(\operatorname{IQR} 7,24)$ & 0.93 \\
\hline Patients with no Known CAD N= 43 & Perfusion defect present $\mathrm{N}=13(30 \%)$ & No perfusion defect $\mathrm{N}=30(70 \%)$ & \\
\hline Age (median) & $69(\mathrm{IQR} 56,73)$ & $54(\mathrm{IQR} 43,67)$ & 0.03 \\
\hline Male & $8(62 \%)$ & $14(47 \%)$ & 0.37 \\
\hline Rest SBP - mean SBP over 3 minutes of adenosine & $-10.3(\mathrm{IQR}-16.67,0)$ & $0(\mathrm{IQR}-7.0,8.5)$ & 0.23 \\
\hline Rest HR - mean HR over 3 minutes (beats per minute) & $16(\operatorname{IQR} 13,23)$ & $21(10,27)$ & 0.46 \\
\hline
\end{tabular}

